\title{
Steganographic Data Heritage Preservation Using Sharing Images App
}

\author{
Zenon Chaczko ${ }^{1}$, Raniyah Wazirali ${ }^{1}$, Lucia Carrion Gordon ${ }^{1}$, \\ Wojciech Bożejko \\ 1 FEIT Faculty of Engineering and IT \\ UTS University of Technology, Sydney, NSW, Australia \\ Zenon.Chaczko@uts.edu.au, \\ Raniyah. Wazirali@uts. edu . au, Lucia.CarrionGordon@student.uts . edu . au \\ 2 Department of Automatics, Mechatronics and Control Systems, \\ Faculty of Electronics, Wrocław University of Science and Technology, Poland \\ wojciech.bozejko@pwr.edu.pl
}

\begin{abstract}
With the advent of smartphones, we have the ability to take a photo and upload it to the internet whenever we desire. Hence, it may be of key importance to include metadata of the image for heritage preservation. This project focuses on heritage concepts and their importance in every evolving and changing digital domain where system solutions have to be sustainable, sharable, efficient and suitable to the basic user needs. Steganography provides a feasible and viable solution to ensure secure heritage preservation of the multimedia content. By embedding information directly into the image, the information about the image will never be lost, as it is not separated from its original source. The aim of the paper is to demonstrate this aspect via an image sharing app that allows users to exchange messages and personalized information that is embedded in the image such that it is inaccessible without knowing their keys.
\end{abstract}

\section{Introduction}

Creating images and uploading them has become easy with the introduction of smart phones. Basic metadata of an image could be considered unsuitable for the purpose of personal information due to its easy access through websites and other applications that can extract metadata from an image. The heritage term is defining as the crucial and central part of the research; we can refer it to 'heritage is those items and places that are valued by the community and is conserved and preserved for future generations [1]. The data is often in isolation. However, the data needs to be with the connections and relationships. It gives the meaning of the information. If that heritage is not preserved, the information can be lost forever. The aim of this research paper demonstrates how steganography can be used for embedding critical data into the image that is readable via a dedicated visualization tools [2], [3]. 


\section{An Overview of Steganographic Sharing}

The development of the preservation framework is related with the value of information and based in the improvement of workflow model. Steganography is the practice of concealing information within another piece of information. This information can later be retrieved by someone with the right key. Figure 1 shows the basic architecture of steganography. The most common way of hiding information within images is by selecting an area using a key provided by the user, and then changing the least significant bits of the color of the pixels in that area to that of the bits of information the user wants to hide. This method is undetectable to the naked eye, and can be difficult to discover even programmatically.

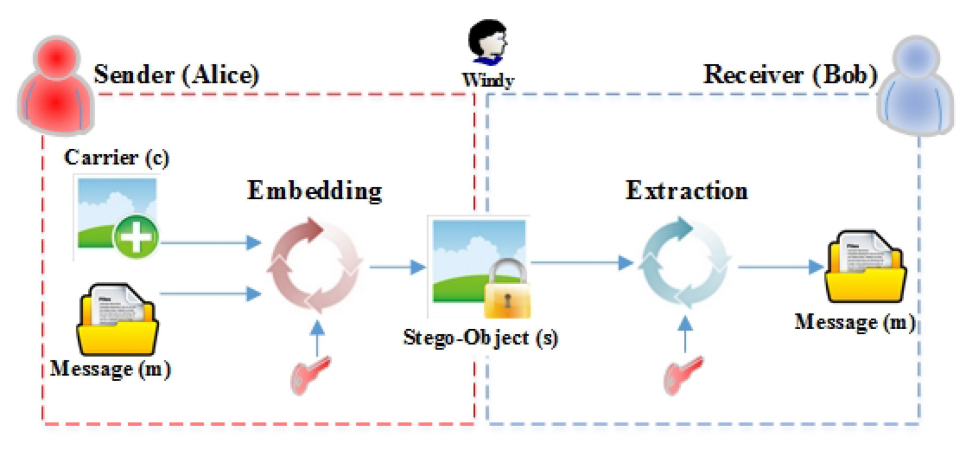

Fig. 1. SDHP Workflow Model

Serendipity is also a process which leads to a serendipitous finding, and here the insight is the key element. Serendipity as a process starts with something unexpected or odd happening - an event, result, encounter or situation/context - that triggers insight. And when this insight will eventually leads to value creation for the individual, community or company, then we are wittnessing serendipity. In the global business world great insights are rare and therefore so valuable. The competitive edge can often be achieved by only one single insight well executed. Therefore understanding serendipity in all forms becomes a vital part of expertise in SDHP.

The images produced by the Photo App will have messages embedded in them, so it's somewhat irrelevant in the context of an image sharing app. Human cultural heritage, documents and artifacts increase regularly and place Data Management as a crucial issue. Figure 2 shows the Steganography Data Heritage Preservation (SDHP) Workflow Model. The first stage involves exploration and approaches based on review of recent advances. The second stage involves adaptation of architectural framework and development of software system architecture in order to build the system prototype. Increasing regulatory compliance mandates are forcing enterprises to seek new approaches to managing reference 


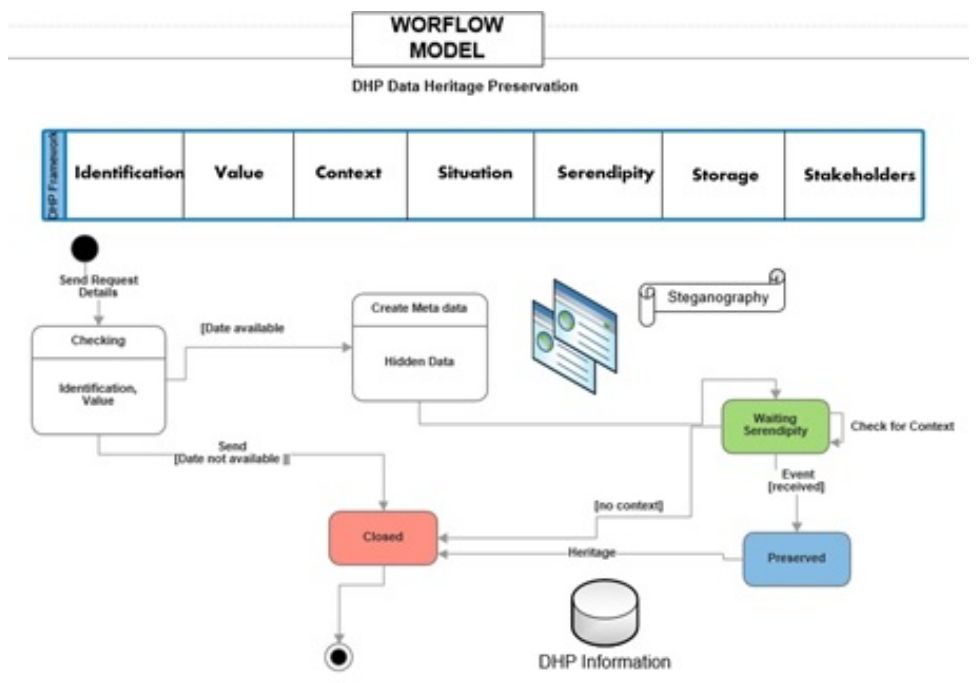

Fig. 2. SDHP Workflow Model

data. The approach of tracking reference data in spreadsheets and doing manual reconciliation can be time consuming and error prone. As various organizations merge and businesses evolve, reference data must be continually mapped and merged as applications are linked and integrated, accuracy and consistency, realize improved data quality, strategy lets organizations adapt reference data as the business evolves. It is important to highlight the type and structure of data. Through the time preserving digital information has a process for designing a practical system for managing massive amounts of critical data. An example of such a process can be found in $[1,5]$. The way to improve the understanding of the methodology, the information has to consider two dimensions: access dimension and cognitive dimension. Both of them have the level of importance in terms of the results. As a methodology of treatment digital preservation, it could be risky even when the strategy could develop a clear idea of digital resources and digital artifacts. Steganography is a powerful and effective multimedia tool for the Digital Heritage Preservation. This can be clearly shown in the presented case study of a successful implementation of steganography technique in the Photo App that allows editing photos, adding messages and uploading the gallery for viewing, exchanging messages and searching for images by other users.

\subsection{Patterns for DHP}

What is heritage conservation? A brief overview Heritage conservation doesn't mean freezing a building in time, creating a museum or tying the hands of property owners so they can't do anything with their properties. Instead, it seeks to maintain and thereby increase the value of buildings by keeping their original built form and architectural elements, favouring their restoration rather 
than replacement and, when restoration is impossible, recreating scale, period and character.

Heritage preservation and designation increases property values, both of the restored building and surrounding properties.

Heritage preservation can be a draw to tourism and helps businesses attract customers. Retaining the historic integrity of a neighbourhood or downtown attracts people just for that ambiance alone and that attracts business. A small town without a heritage main street attracts no one.

Restoration reduces construction and demolition waste and uses less than half the energy of new construction. Heritage preservation is an investment in our community that rewards us today and leaves an invaluable resource for future generations.

Deep learning (deep machine learning, or deep structured learning, or hierarchical learning, or sometimes DL) is a branch of machine learning based on a set of algorithms that attempt to model high-level abstractions in data by using multiple processing layers with complex structures, or otherwise composed of multiple non-linear transformations.

Deep learning is part of a broader family of machine learning methods based on learning representations of data. An observation (e.g., an image) can be represented in many ways such as a vector of intensity values per pixel, or in a more abstract way as a set of edges, regions of particular shape, etc.. Some representations make it easier to learn tasks (e.g., face recognition or facial expression recognition) from examples. One of the promises of deep learning is replacing handcrafted features with efficient algorithms for unsupervised or semi-supervised feature learning and hierarchical feature extraction.

Research in this area attempts to make better representations and create models to learn these representations from large-scale unlabeled data. Some of the representations are inspired by advances in neuroscience and are loosely based on interpretation of information processing and communication patterns in a nervous system, such as neural coding which attempts to define a relationship between various stimuli and associated neuronal responses in the brain.[3]

Various deep learning architectures such as deep neural networks, convolutional deep neural networks, deep belief networks and recurrent neural networks have been applied to fields like computer vision, automatic speech recognition, natural language processing, audio recognition and bioinformatics where they have been shown to produce state-of-the-art results on various tasks.

\subsection{Steganographic Data Heritage App}

Steganography provides a feasible and viable solution to these problems. By embedding information directly into the image, the information about the image will not be lost, if it is separated from its original source. The aim of the capstone is to demonstrate this aspect via an image sharing app that allows users to exchange messages and personalized information that is embedded in the image such that it is inaccessible without knowing their keys, and metadata that can be used by search engines to categorize the image and its contents. The developed 
application will provide a platform for users to create an account, share images, embed information within the image, and search for images other users have uploaded. This will allow great opportunity for data heritage through embedding the metadata to the multimedia. Therefore, the metadata can travel from software to another without any lost.

The aim of this case study is to create an image sharing application that shows the power of using steganography in multimedia. There are a number of features aimed at both the user taking photos, and the user viewing them. Users will be able to create accounts, post pictures, put comments with the pictures, aimed at general or specific users, search posted photos, and share posted photos.

This case study seeks to develop and implement a method of embedding multiple steganographically hidden messages within a single image. While there are methods to do this out there currently, they can only store a limited number of messages. This project aims to create a method scalable to potentially use all of the applicable space in the image to store different messages.

This project also encompasses an application using the steganographical method we will create. This will provide a working example of how this method can be used in the real world, with some additional features such as a search engine.

\subsection{Values in Context}

The search for values and meaning has become a pressing concern.[2]

The issue is what to conserve and how to conserve. Values are an important determining factor in the current practices and future prospects of the conservation field. The capacity of the conservation field to enrich cultural life and the visual arts in societies worldwide. Try to understand the processes- specific and general - by which material heritage conservation functions in the context of modern society, to look at the kinds of social and cultural dynamics making the greatest impact on conservation's role in society, presently and in the future; and to consider ideas, concepts, and research themes study.

\section{$3 \quad$ Future Projects}

Data preservation: Digitalization of the Heritage, the result of proposal is to have like a result of the experimental work, a reliable Framework for measure the digital age of the information and patterns that qualified usability and accessibility of the data. The best pathway for commercialization could be some of them.

- Commercial Business Structure like a Partnership assuming the cost of the investment and the taxes that generate the buying of the equipment for implementation of the scanning in the digitalization.

- Initial Public Offering IPO, because the application of the data preservation could be focus on Entities from Government and Historical materials and artifacts that sometimes have to be preserved with a public responsibility. 


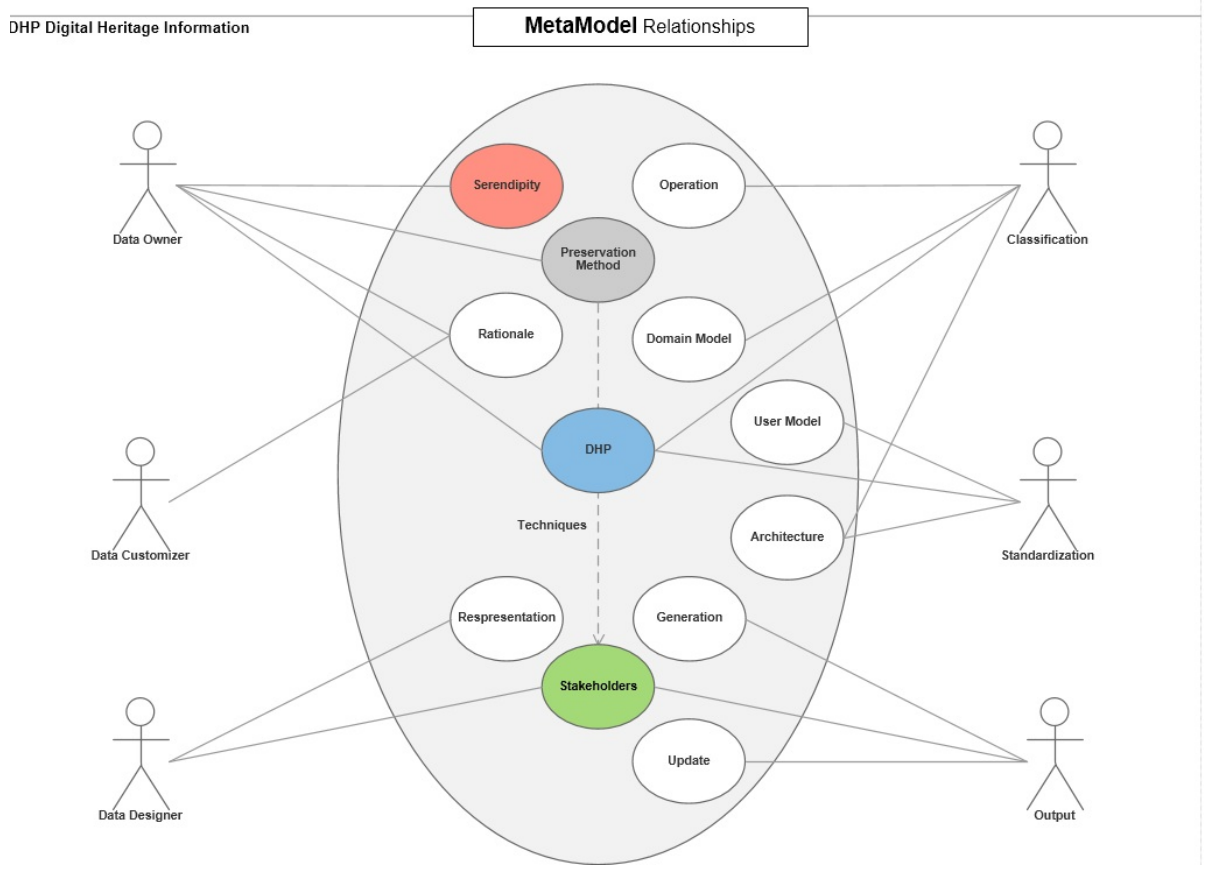

Fig. 3. DHP Relationships

- This research could have through the market with POCs proof of concepts, showing the advantages and challenges of the new solution. In this case the relationship between the process and the final patterns there is a model.

\section{Conclusion}

The context, relation and situation of the Serendipitous Heritage are impressive relevant in the research because it gives the sense of the future of the Knowledge in the World. Through the Socio - Technical, Cultural fields, the process of Preservation will do a contribution for more research. The use of tools and techniques like Steganography, the concepts of Software Architecture will have a real approach and meaninful characteristics for the relevance of the investigation. Steganographic photo sharing app was fairly successful in implementing its goals. It clearly shows the power of steganography as a useful multimedia tools. The Photo App fulfilled all of the Software Requirement Specifications, and a demonstrated ways in which steganography can be utilised outside simple secret messaging. 


\section{References}

1. Bożejko W., Hejducki Z., Wodecki M., Applying metaheuristic strategies in construction projects management, Journal of Civil Engineering and Management, Volume 18, Issue 5, 621-630 (2012)

2. Challa S., Gulrez T., Chaczko Z., Paranesha T.N.: Opportunistic information fusion: a new paradigm for next generation networked sensing systems. in: Information Fusion, 8th International Conference on, vol. 1, 720-727 (2005)

3. Gulden, J.: Methodical support for model-driven software engineering with enterprise models. Universität Duisburg-Essen 1-332 (2013)

4. Johnson N.F., Jajodia S.: Exploring steganography: Seeing the unseen, Computer vol. 31, no. 2, 26-34 (1998)

5. Smutnicki C., Bożejko W., Parallel and Distributed Metaheuristics, in: Conference Computer Aided Systems Theory EUROCAST 2015 (Roberto Moreno-Díaz, Franz Pichler, Alexis Quesada-Arencibia, eds.), Lecture Notes in Computer Science Vol. 9520, 72-79 (2015)

6. UNESCO: Information Document Glossary of World Heritage Terms (1996), http://whc.unesco.org/archive/gloss96.htm

7. Wazirali R. R., Chaczko Z., Kale A.: Digital Multimedia Archiving Based on Optimization Steganography System. Asia-Pacific Conference on Computer Aided System Engineering (APCASE 2014). IEEE Press, 82-86 (2014) 